\title{
Alterações ocorridas pelo manejo em Latossolo, Rondônia, Amazônia Brasileira
}

\author{
Gustavo Souza Valladares ( $\left.{ }^{*}\right)$; Mateus Batistella $\left({ }^{2}\right)$; Marcos Gervasio Pereira $\left({ }^{3}\right)$ \\ (') Universidade Federal do Ceará (UFC), Departamento de Ciências do Solo, Caixa Postal 12168, 60021-970 Fortaleza (CE). \\ (2) Embrapa Monitoramento por Satélite, Av. Soldado Passarinho, 303, 13070-115 Campinas (SP). \\ (3) Universidade Federal Rural do Rio de Janeiro (UFRRJ), BR 465 km 7, 23890-000 Seropédica (RJ). \\ (*) Autor correspondente: valladares@ufc.br
}

Recebido: 28/jul./2010; Aceito: 6/dez./2010

\begin{abstract}
Resumo
O objetivo deste trabalho foi avaliar atributos de fertilidade, frações da matéria orgânica, incluindo ácido fúlvico, ácido húmico, humina e matéria orgânica leve, densidade e volume total de poros, em um Latossolo Amarelo distrófico, sob cultura perene, pastagem e floresta amazônica em área de assentamento rural conhecida como Gleba Machadinho (RO). As coletas das amostras de solo ocorreram em 2004; foram amostradas as camadas superficiais do solo na profundidade de 0-0,1 m. Verificou-se melhoria na fertilidade dos solos sob culturas perenes, com diminuição da acidez e aumento dos teores de nutrientes. Não foi verificada diferença nos teores totais de carbono orgânico dos solos, porém variações nas frações orgânicas, ocorrendo menores teores de matéria orgânica leve e maior relação das substâncias húmicas alcalino solúveis em comparação à humina nos solos com culturas perenes. Nas áreas de pastagem, o pisoteio animal causou aumento da densidade do solo e redução do volume total de poros.
\end{abstract}

Palavras-chave: manejo do solo, solos da Amazônia, propriedades dos solos.

\section{Changes promoted by management in Oxisol, Rondônia, Brazilian Amazon}

\section{Abstract}

This article evaluates fertility attributes, such as density, total volume of pores, and fractions of organic matter, including fulvic acid, humic acid, and soft organic matter, in a distrophic Oxisol, under perennial agriculture, pasture, and forest, in an Amazonian rural settlement called Machadinho d'Oeste, Rondônia. Fieldwork was done in 2004 when superficial soil horizons were sampled at depths between the soil surface and $0.1 \mathrm{~m}$. Soil fertility increases under perennial agriculture, with lower acidity and higher amounts of nutrients. No significant difference was observed in soil organic carbon. However, variations in organic fractions were due to lower levels of soft organic matter and stronger relationships between soluble alkaline humic substances in comparison with soil humines under perennial agriculture. In areas of pasture, animal movement increased soil density and decreased the total volume of pores.

Key words: soil management, Amazon soils, soil properties.

\section{INTRODUÇÃO}

Nos últimos anos é crescente a preocupação com o desflorestamento e a degradação dos solos da Amazônia (Alves et al., 1999; Muller et al., 2001; Brondizio et al., 2002; Batistella e Moran, 2005; Fearnside, 2006). O manejo inadequado desses solos pode levar à degradação por erosão (Lu et al., 2004) e perdas de nutrientes em solos naturalmente de baixa fertilidade, fenômenos favorecidos pelos elevados índices pluviométricos verificados na região, de $2.354 \mathrm{~mm}$ (VAlladAres et al., 2003a). As grandes áreas desflorestadas para formação de pastagens ou lavouras podem gerar declínio nos estoques de carbono e nitrogênio de origem orgânica (Freixo et al., 2002; Leite et al., 2003; Torres et al., 2005).
A redução da produtividade em lavouras da Amazônia tem sido atribuída à baixa reserva de nutrientes, elevada acidez, alta fixação de fósforo, redução da porosidade e da infiltração de água (Anjos et al., 1994; Souza et al., 2004). Estes aspectos justificam o manejo conservacionista dos solos desde o início de seu uso agrícola (Muller et al., 2001).

A matéria orgânica do solo, interagindo com a fração mineral, interfere na dinâmica de nutrientes, favorecendo a manutenção da fertilidade do solo, como também das propriedades físicas e biológicas (MENDONZA et al., 2000; Fontana et al., 2006), sendo muito importante em solos intemperizados, como os Latossolos da Amazônia. Nesse contexto, o teor da matéria orgânica no solo e das substâncias húmicas tem sido utilizado como indicadores 
de qualidade dos solos em razão de sua interação com a fração mineral e por refletirem o manejo dos solos (SiLva et al., 2006; Fontana et al., 2006; Grego et al., 2007).

Este trabalho teve como objetivo avaliar atributos de fertilidade do solo, densidade do solo, porosidade total e as fraçōes húmicas em um Latossolo Amarelo distrófico, sob diferentes usos e coberturas na Amazônia.

\section{MATERIAL E MÉTODOS}

O trabalho foi realizado com amostras coletadas na área de assentamento rural denominada Gleba Machadinho (Figura 1), nos municípios de Machadinho d'Oeste e Vale do Anari (RO), situada entre as coordenadas $9^{\circ} 15^{\prime}$ e 9॰48' de latitude sul e 6148' e 62³0' de longitude oeste. A área faz parte da bacia hidrográfica do rio Machadinho, afluente do Rio Machado ou Ji-Paraná.

$\mathrm{Na}$ região de Machadinho d'Oeste, o clima pode ser classificado como Am segundo classificaçáo de Köppen, o que indica temperaturas elevadas durante todo o ano (megatérmico) com pequena deficiência hídrica na estação seca do ano, sendo este o tipo climático que predomina na região Amazônica (Nimer, 1989; VALLADAREs et al., 2003a). O solo estudado foi classificado como Latossolo Amarelo distrófico, com cores do matiz 7,5YR ou mais amarelo no horizonte B latossólico, com horizonte A moderado, raramente com espessura superior a $0,15 \mathrm{~m}$ e textura variando de média a argilosa (Valladares et al., 2003a; EMBrapa, 2006). Na tabela 1 verifica-se os atributos sobre a fertilidade e a granulometria dos solos estudados.

Foram amostrados epipedons na profundidade de 0-0,1 $\mathrm{m}$, sendo quatro repetiçóes sob vegetação nativa, 11 repetiçôes sob pastagens diversas, principalmente com Brachiaria brizantha (Hochst. Ex A. Rich) Staf e seis repetiçóes sob culturas perenes de Coffea arabica (Lineu) e Paullinia cupana Kunth (Guaraná). Selecionaram-se pontos amostrais em posiçóes distintas da paisagem: topo ou terços superiores com declividades entre $0-5 \%$, com objetivo de se eliminar efeitos de variaçôes do relevo.

Em amostras indeformadas foi determinada a densidade do solo (Ds) e a densidade das partículas, e através destas estimou-se a porosidade total (VTP). Nas amostras deformadas coletadas com auxílio de trado foi caracterizada a fertilidade do solo por meio de determinaçáo de $\mathrm{pH}$ em água, $\mathrm{pH}$ em $\mathrm{CaCl}_{2}$, bases trocáveis, alumínio, hidrogênio, saturação por bases, fósforo, cobre, manganês, ferro, zinco e boro, conforme (ЕMBrapa, 1997), carbono orgânico $(\mathrm{C})$, fração ácido fúlvico (FAF), fração ácido húmico (FAH), humina (HUM) e matéria orgânica leve (MOL) conforme metodologias adaptadas e descritas em Benites et al. (2003) e Fontana et al. (2006).

Os dados foram analisados estatisticamente por análise de variância e teste de Tukey a 5\% e 10\% de significância e por análise de correlação de Pearson.

\section{RESULTADOS E DISCUSSÃO}

Os valores de $\mathrm{pH}$ em água variaram de 4,9 a 5,7 e em $\mathrm{CaCl}_{2}$ de 4,1 a 4,9. Através dos valores de $\mathrm{pH}$ em água e em $\mathrm{CaCl}_{2}$, foi verificada diferença entre os usos das terras, sendo os valores mais elevados para as culturas perenes quando comparados à vegetação nativa (Tabela 2 ). Os valores $\mathrm{pH}$ em $\mathrm{CaCl}_{2}$ foram maiores na área de pastagem quando em relação à vegetação nativa. Os valores observados estáo um pouco acima da média dos verificados para os Latossolos da área de estudo, conforme aponta a literatura (VAlladares et al., 2003a). Este resultado é reflexo de calagens efetuadas por alguns agricultores da região (Grego et al., 2007). Os teores de $\mathrm{H}^{+}$e de $\mathrm{Al}^{3+}$ tiveram a mesma tendência dos valores de $\mathrm{pH}$, com diferença estatística significativa nos solos sob vegetação nativa, com valores superiores aos solos sob culturas perenes e pastagens. Mesmo sob vegetação nativa os teores de $\mathrm{Al}^{3+}$ não foram considerados elevados, valor médio de $0,8 \mathrm{cmol}_{\mathrm{c}} \mathrm{kg}^{-1}$, e nas áreas de cultura perene e pastagens os teores médios de $0,2 \mathrm{cmol}_{\mathrm{c}} \mathrm{kg}^{-1}$ náo indicam toxidez pelo elemento. Verificam-se altas correlaçóes positivas dos valores de $\mathrm{pH}$ em água e $\mathrm{CaCl}_{2}$ entre si e os atributos $\mathrm{Ca}^{2+}$, $\mathrm{Mg}^{2+}$ e V\%, e altas correlaçốes negativas com $\mathrm{Al}^{3+}$ e $\mathrm{H}^{+}$ (Tabela 3). As correlaçôes entre $\mathrm{Al}^{3+} \mathrm{e} \mathrm{H}^{+}$também foram positivas e altas.

Os valores de saturação por bases (V\%) foram superiores nas áreas com culturas perenes, com média de $42 \%$ em comparação com as áreas de vegetação nativa, com V\% de $14 \%$. A saturação por bases proporcionou correlaçôes positivas com os teores de $\mathrm{Ca}^{2+} \mathrm{e} \mathrm{Mg}^{2+}$ e negativas com $\mathrm{Al}^{3+}$ e $\mathrm{H}^{+}$.

Com relação aos teores de $\mathrm{Ca}^{2+}$, as áreas com culturas perenes também têm valores mais elevados deste nutriente em comparação às áreas de mata nativa, com valores entre 0,7 e 2, $4 \mathrm{cmol}_{\mathrm{c}} \mathrm{kg}^{-1}$. Para os teores de $\mathrm{Mg}^{2+}, \mathrm{K}^{+}$e $\mathrm{Na}^{+}$e P não foram verificadas diferenças entre as áreas estudadas. Os teores de $\mathrm{Ca}^{2+} \mathrm{e} \mathrm{Mg}^{2+}$ tiveram correlação positiva entre si. Os baixos teores de $\mathrm{P}$ podem ser reflexo da natureza dos solos estudados que possuem alta capacidade de adsorção de P (Valladares et al., 2003b), pois são muito intemperizados, com presença de óxidos e oxi-hidróxidos de ferro e alumínio, altos teores de argila e muitos solos possuem caráter mesoférrico (VALLADARES et al., 2003b), apesar das cores amareladas.

Os micronutrientes possuem comportamento diferenciado, para os teores de $\mathrm{Cu}, \mathrm{Zn}$ e $\mathrm{B}$ não sendo verificada diferença significativa entre as diferentes áreas. Os teores de Fe foram significativamente superiores nas áreas de pastagem em comparação às áreas com culturas perenes. O maior teor de $\mathrm{Mn}$ ocorreu nas áreas de culturas perenes.

Quanto aos teores de C, os valores variaram entre 20 e $26 \mathrm{~g} \mathrm{~kg}^{-1}$, não havendo diferença entre as áreas. As pastagens parecem promover o aumento do teor de $\mathrm{C}$ no solo 
(D’AndréA et al., 2004; Fontana et al., 2006). Alguns trabalhos com solos da Amazônia demonstram que, logo após o desflorestamento ocorre a reduçáo dos teores de C no solo (Cerri et al., 2003; Moreira e Malavolta, 2004), porém após alguns anos com pastagem o carbono perdido é recuperado, podendo haver aumento de até $54 \%$ do estoque em comparação com a floresta inicial (Bernoux et al., 1999; Cerri et al., 2003; Silva et al., 2006). Os teores de $\mathrm{C}$ promoveram correlaçáo positiva com a FAH, HUM e MOL, sendo os valores de correlação mais elevados para a MOL (Tabela 3).
A fração da matéria orgânica humificada predominante foi a HUM, seguida da FAF e da FAH para todos os usos considerados. Para nenhuma das fraçóes foi observada diferença entre os tratamentos. Valores mais elevados de HUM podem indicar maior tamanho das moléculas e maior grau de estabilidade desta fração, que revela natureza química essencialmente alifática, proveniente da preservação seletiva de biopolímeros oriundos de plantas e microrganismos que interagem intimamente com a fração mineral dos solos (Rice, 2001). A natureza oxídica da fração mineral e a alternância de períodos chuvosos

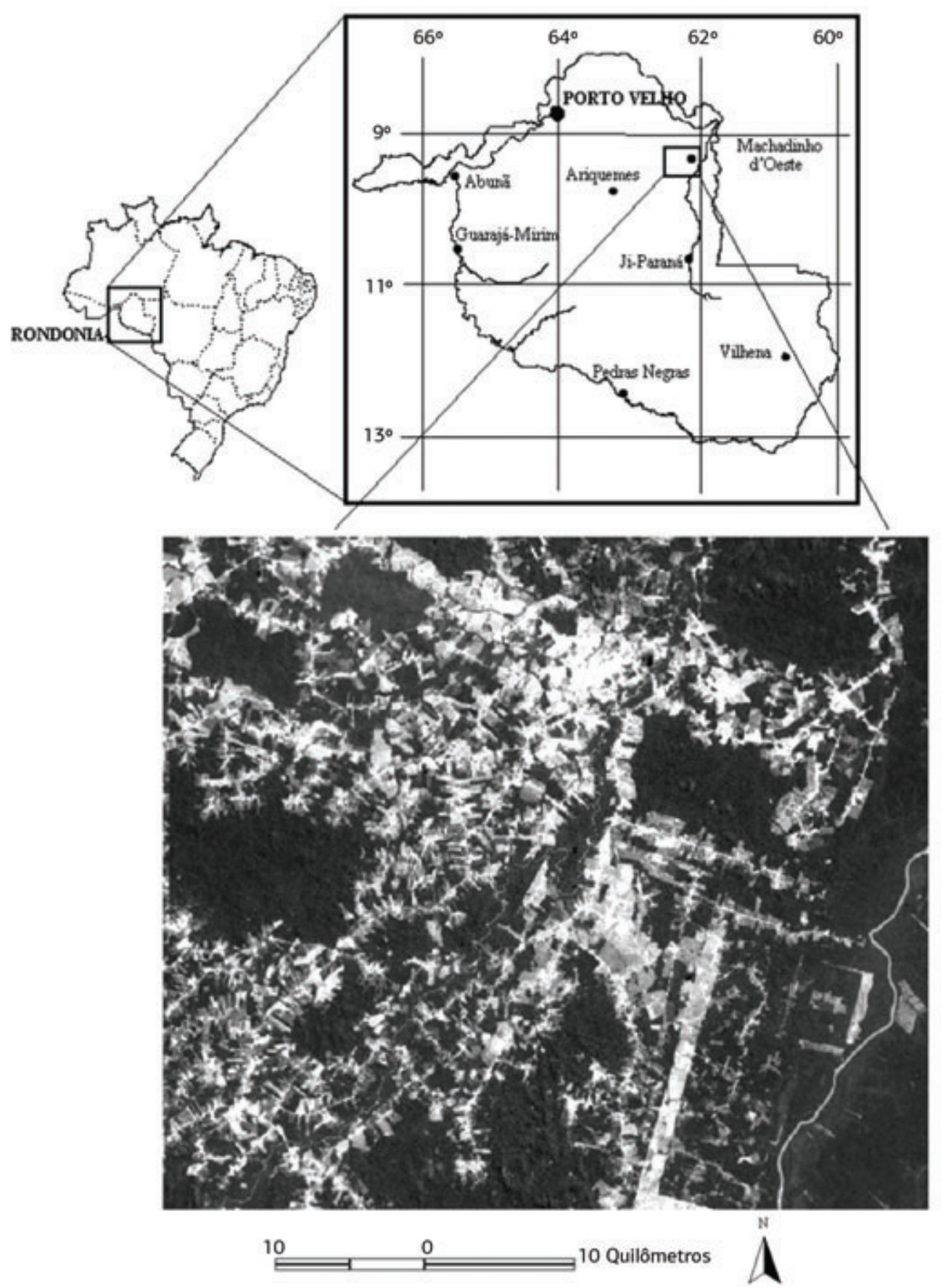

Figura 1. Localização da Gleba Machadinho (RO). 
Tabela 1. Valores médios, máximos e mínimos de atributos da camada superficial dos Latossolos Amarelos estudados

\begin{tabular}{|c|c|c|c|c|c|c|c|c|}
\hline \multirow[t]{2}{*}{ Medida } & \multirow[t]{2}{*}{ pH água } & Valor S & $\mathrm{H}^{+}+\mathrm{Al}^{3+}$ & Valor T & \multirow{2}{*}{$\begin{array}{c}\text { Valor V } \\
\%\end{array}$} & Argila & Silte & Areia \\
\hline & & & $\mathrm{cmol}_{\mathrm{c}} \mathrm{kg}^{-1}$ & & & \multicolumn{3}{|c|}{$\mathrm{g} \mathrm{kg}^{-1}$} \\
\hline Média & 5,4 & 2,3 & 5,0 & 7,3 & 30 & 371 & 244 & 385 \\
\hline Máximo & 6,6 & 5,9 & 8,0 & 8,6 & 67 & 520 & 360 & 710 \\
\hline Mínimo & 4,7 & 0,4 & 2,8 & 6,0 & 6 & 200 & 80 & 150 \\
\hline
\end{tabular}

S: soma de Bases, T: capacidade de troca catiônica do solo, V: Saturação por bases

Tabela 2. Valores médios dos atributos na profundidade de 0-0,10 $\mathrm{m}$ dos Latossolos Amarelos estudados sob diferentes usos da terra

\begin{tabular}{|c|c|c|c|}
\hline Atributo & $\begin{array}{c}\text { Vegetação } \\
\text { nativa }\end{array}$ & $\begin{array}{l}\text { Cultura } \\
\text { perene }\end{array}$ & Pastagem \\
\hline pH em água & 4,9 B & 5,7 A & $5,5 \mathrm{AB}$ \\
\hline $\mathrm{pH}$ em $\mathrm{CaCl}_{2}$ & $4,1 \mathrm{~B}$ & $4,9 \mathrm{~A}$ & $4,6 \mathrm{~A}$ \\
\hline $\mathrm{Al}^{3+}\left(\mathrm{cmol}_{\mathrm{c}} \mathrm{kg}^{-1}\right)$ & $0,8 \mathrm{~A}$ & $0,2 \mathrm{~B}$ & $0,2 \mathrm{~B}$ \\
\hline $\mathrm{H}^{+}\left(\mathrm{cmol}_{\mathrm{c}} \mathrm{kg}^{-1}\right)$ & $6,2 \mathrm{~A}$ & $4,1 \mathrm{~B}$ & $4,6 \mathrm{~B}$ \\
\hline $\mathrm{Ca}^{2+}\left(\mathrm{cmol}_{\mathrm{c}} \mathrm{kg}^{-1}\right)$ & $0,7 \mathrm{~B}$ & $2,4 \mathrm{~A}$ & $1,7 \mathrm{AB}$ \\
\hline $\mathrm{Mg}^{2+}\left(\mathrm{cmol}_{c} \mathrm{~kg}^{-1}\right)$ & $0,3 \mathrm{~A}$ & $0,6 \mathrm{~A}$ & $0,5 \mathrm{~A}$ \\
\hline $\mathrm{K}^{+}$(cmolc kg-1) & $0,12 \mathrm{~A}$ & $0,18 \mathrm{~A}$ & $0,12 \mathrm{~A}$ \\
\hline V (\%) & $14 \mathrm{~B}$ & $42 \mathrm{~A}$ & $32 \mathrm{AB}$ \\
\hline $\mathrm{P}\left(\mathrm{mg} \mathrm{L}^{-1}\right)$ & $2,5 \mathrm{~A}$ & $2,0 \mathrm{~A}$ & $1,9 \mathrm{~A}$ \\
\hline $\mathrm{Cu}\left(\mathrm{mg} \mathrm{L}^{-1}\right)$ & $0,58 \mathrm{~A}$ & $0,46 \mathrm{~A}$ & $0,73 \mathrm{~A}$ \\
\hline $\mathrm{Fe}\left(\mathrm{mg} \mathrm{L}^{-1}\right)$ & $196 \mathrm{AB}$ & $78 \mathrm{~B}$ & $306 \mathrm{~A}$ \\
\hline $\mathrm{Mn}\left(\mathrm{mg} \mathrm{L}^{-1}\right)$ & $7,5 \mathrm{~B}$ & $47,3 \mathrm{~A}$ & $18,3 \mathrm{AB}$ \\
\hline $\mathrm{Zn}\left(\mathrm{mg} \mathrm{L}^{-1}\right)$ & $3,8 \mathrm{~A}$ & $3,4 \mathrm{~A}$ & $4,9 \mathrm{~A}$ \\
\hline $\mathrm{B}\left(\mathrm{mg} \mathrm{L}^{-1}\right)$ & $0,13 \mathrm{~A}$ & $0,24 \mathrm{~A}$ & $0,18 \mathrm{~A}$ \\
\hline $\mathrm{C}\left(\mathrm{mg} \mathrm{kg}^{-1}\right)$ & $26,0 \mathrm{~A}$ & $20,0 \mathrm{~A}$ & $26,0 \mathrm{~A}$ \\
\hline HUM (mg kg-1) & $7,3 \mathrm{~A}$ & $7,6 \mathrm{~A}$ & $7,8 \mathrm{~A}$ \\
\hline $\mathrm{FAH}\left(\mathrm{mg} \mathrm{kg}^{-1}\right)$ & $2,6 \mathrm{~A}$ & $3,4 \mathrm{~A}$ & $2,7 \mathrm{~A}$ \\
\hline FAF $\left(\mathrm{mg} \mathrm{kg}^{-1}\right)$ & $4,2 \mathrm{~A}$ & $4,2 \mathrm{~A}$ & $3,5 \mathrm{~A}$ \\
\hline $\mathrm{MOL}\left(\mathrm{mg} \mathrm{kg}^{-1}\right)$ & $11,9 \mathrm{~A}$ & $5,0 \mathrm{~B}$ & $12,4 \mathrm{~A}$ \\
\hline $\mathrm{FAH} / \mathrm{FAF}$ & $0,62 \mathrm{~A}$ & $0,81 \mathrm{~A}$ & $0,77 \mathrm{~A}$ \\
\hline EA/HUM & $0,95 \mathrm{AB}$ & $1,00 \mathrm{~A}$ & $0,79 \mathrm{~B}$ \\
\hline Ds $\left(\mathrm{Mg} \mathrm{m}^{-3}\right)$ & 0,94 B & $1,06 \mathrm{AB}$ & 1,17 A \\
\hline VTP (\%) & $58,5 \mathrm{~A}$ & $52,8 \mathrm{AB}$ & 49,1 B \\
\hline
\end{tabular}

Letras diferentes na mesma linha indicam diferença estatística significativa pelo teste de Tukey a 5\% e a 10\%. HUM-humina, FAH-fraçáo ácido húmico, FAF-fração ácido fúlvico, MOL-matéria orgânica leve, EA=FAF+FAH, Ds-densidade do solo, VTP, volume total de poros.

e de estiagem contribuem na intensificação da formação de humina em solos tropicais (DABIN, 1981). Nos solos estudados a fração HUM representa menos que $45 \%$ do C orgânico total do solo (Figura 2), indicando baixo grau de evolução do húmus, o que segundo Canellas et al. (2003) é reflexo de ambientes com restriçóes fortes à atividade microbiana. Devido à boa drenagem e à alta pressão de oxigênio, as vias de humificação prováveis (CAMARGo et al., 1999) são a de insolubilização (humificação dos compostos fenólicos solúveis) e neossíntese microbiana (humificação das moléculas simples através da síntese microbiana).

As fraçôes FAF e FAH, por conter menor massa molecular, são menos estáveis, podendo ser facilmente mineralizadas, translocadas para camadas subsuperficiais ou polimerizadas, diminuindo seus teores nos solos. Alguns trabalhos indicam maiores teores da fraçáo HUM nos solos
(Rosales et al., 1999; Fontana et al., 2006; Passos et al., 2007). Miranda et al. (2007) trabalhando com solos do Estado do Rio de Janeiro também verificaram predomínio da fração humina, sendo a proporção desta fração ainda maior em solos agrícolas.

Os teores da fraçáo humina variaram de 7,3 a $7,8 \mathrm{~g} \mathrm{~kg}^{-1}$, os da FAF de 3,5 a 4,2 $\mathrm{g} \mathrm{kg}^{-1}$ e os da FAH de 2,6 a $3,4 \mathrm{~g} \mathrm{~kg}^{-1}$ (Tabela 2). Os valores da relação FAH/ FAF foram baixos e inferiores a 1 , indicando favorecimento da degradação das fraçóes mais estáveis ou desfavorecendo sua formação, mas nota-se que este resultado não é exclusivo do manejo agrícola dos solos, uma vez que o mesmo comportamento foi observado nas áreas de vegetaçáo nativa. Estes resultados são comuns em solos tropicais, indicando baixas taxas de humificação e altas de mineralização da matéria orgânica, sendo reflexo do baixo teor de bases, da acidez dos solos e restrição da atividade microbiana (Miranda et al., 2007).

A soma da FAF e da FAH representa o extrato alcalino (EA). A relação EA/HUM apresentou valores de 0,79 na pastagem, 0,95 na vegetação nativa e 1,00 nas culturas perenes, sendo verificada diferença estatística significativa entre as áreas de culturas perenes e a de pastagem. Estes resultados demonstram que nas pastagens as fraçōes menos estáveis estão proporcionalmente menores do que a HUM, o que pode ser reflexo de maior modificação nesses ambientes (CANellas et al., 2003; Fontana et al., 2006) favorecendo sua mineralização ou até mesmo a translocação. A FAF e a FAH tiveram baixa correlação positiva entre si, e a FAH revelou maiores valores de correlação positiva com a HUM (Tabela 3).

Quanto à MOL os valores desta fração foram significativamente maiores nas áreas de vegetação nativa $\left(11,9 \mathrm{~g} \mathrm{~kg}^{-1}\right)$ e pastagem $\left(12,4 \mathrm{~g} \mathrm{~kg}^{-1}\right)$ em comparação com as áreas de culturas perenes $\left(5,0 \mathrm{~g} \mathrm{~kg}^{-1}\right)$. Nas áreas de vegetação nativa é comum o elevado aporte de material orgânico vegetal oriundo da queda de folhas, galhos, frutos e outras partes vegetais, como também de elevada densidade de raízes, favorecendo o acúmulo de MOL na camada superficial do solo. Nas áreas de pastagem, a elevada densidade de raízes fasciculadas, que possuem altos teores de lignina também favorece os maiores teores de MOL. Nas áreas com culturas perenes, menor densidade de raízes e menor aporte de material orgânico de vegetais senescentes, são fatores que conduziram a menores teores de MOL. Estes mesmos fatores explicam a menor relação $\mathrm{MOL} / \mathrm{C}$ nas áreas de culturas perenes 
Tabela 3. Coeficientes de correlação de Pearson dos atributos dos Latossolos Amarelos estudados

\begin{tabular}{|c|c|c|c|c|c|c|c|c|c|c|c|c|}
\hline & $\mathrm{pHCaCl}_{2}$ & pHágua & C & FAF & FAH & HUM & MOL & K & $\mathrm{Ca}$ & Mg & Al & $\mathbf{H}^{+}$ \\
\hline $\mathrm{pHCaCl}_{2}$ & 1.00 & - & - & - & - & - & - & - & - & - & - & - \\
\hline pHágua & 0.98 & 1.00 & - & - & - & - & - & - & - & - & - & - \\
\hline C & -0.09 & -0.05 & 1.00 & - & - & - & - & - & - & - & - & - \\
\hline FAF & -0.12 & -0.06 & 0.22 & 1.00 & - & - & - & - & - & - & - & - \\
\hline FAH & 0.25 & 0.32 & 0.64 & 0.48 & 1.00 & - & - & - & - & - & - & - \\
\hline HUM & 0.36 & 0.42 & 0.66 & 0.43 & 0.70 & 1.00 & - & - & - & - & - & - \\
\hline MOL & -0.24 & -0.23 & 0.92 & -0.07 & 0.34 & 0.37 & 1.00 & - & - & - & - & - \\
\hline $\mathrm{K}^{+}$ & 0.17 & 0.12 & -0.12 & -0.11 & -0.13 & -0.14 & -0.07 & 1.00 & - & - & - & - \\
\hline $\mathrm{Ca}^{2+}$ & 0.86 & 0.88 & -0.01 & 0.11 & 0.48 & 0.55 & -0.27 & 0.02 & 1.00 & - & - & - \\
\hline $\mathrm{Mg}^{2+}$ & 0.68 & 0.68 & 0.05 & -0.08 & 0.38 & 0.37 & -0.11 & 0.08 & 0.78 & 1.00 & - & - \\
\hline $\mathrm{Al}^{3+}$ & -0.78 & -0.72 & 0.29 & 0.27 & 0.02 & -0.11 & 0.35 & -0.21 & -0.57 & -0.50 & 1.00 & - \\
\hline $\mathrm{H}^{+}$ & -0.95 & -0.92 & 0.18 & 0.19 & -0.22 & -0.31 & 0.32 & -0.18 & -0.85 & -0.74 & 0.87 & 1.00 \\
\hline V & 0.93 & 0.93 & -0.06 & -0.03 & 0.39 & 0.46 & -0.27 & 0.16 & 0.96 & 0.85 & -0.71 & -0.94 \\
\hline
\end{tabular}

Valores em negrito são significativos a 5\% de probabilidade. HUM-humina, FAH-fração ácido húmico, FAF-fração ácido fúlvico, MOL-matéria orgânica leve.

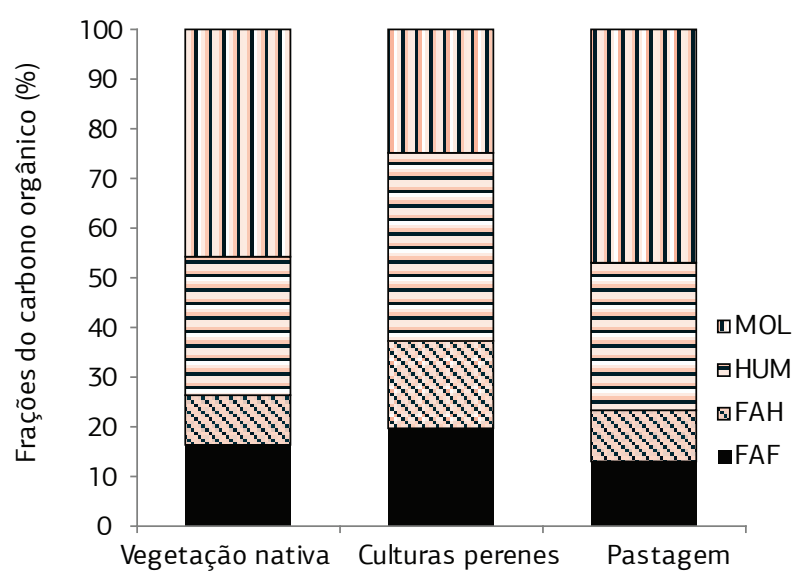

Figura 2. Distribuição percentual das frações do carbono orgânico do solo nos usos e nas coberturas da terra estudados. FAF-fração ácido fúlvico; FAH-fração ácido húmico; HUM-humina; MOLmatéria orgânica leve.

$(0,25)$ em comparação com as áreas de vegetação nativa $(0,45)$ e pastagem $(0,43)$. Estes resultados indicam maior proporção da matéria orgânica humificada nos solos estudados, resultados que são corroborados por Fontana et al. (2006). Já Miranda et al. (2007) observaram nas camadas superficiais dos solos proporção de matéria orgânica humificada inferior a 50\%. A menor proporção de MOL nas áreas de cultura perene pode ser reflexo da maior fertilidade natural e menor acidez dos solos sob culturas perenes, condiçóes que desfavorecem o acúmulo de MOL (Cerri e Volkof, 1988; Tomé Júnior, 1997; Miranda et al., 2007; Rangel e Silva, 2007).

Os valores de densidade do solo foram de 0,94, 1,06 e $1,17 \mathrm{Mg} \mathrm{m}^{-3}$ e de VTP de 58,5\%, 52,8\% e 49,1\%, respectivamente, para a área de vegetação nativa, culturas perenes e pastagem, sendo verificada diferença entre a vegetação nativa e as áreas de pastagem para estes dois atributos físicos. Estes resultados indicam que o pisoteio do gado promoveu compactação na camada superficial do solo, o que pode favorecer a degradação dos solos. Resultados semelhantes foram observados (Muller et al., 2001; Giarola et al., 2007; Valladares et al., 2007) em diferentes regióes edafoclimáticas. A redução do VTP nas pastagens deve ser um reflexo da redução da macroporosidade, uma vez que a microporosidade náo parece ser influenciada diretamente pelo manejo no solo (Giarola et al., 2007; VAlladares et al., 2007).

\section{CONCLUSÕES}

Os solos com culturas perenes são propícios a melhorias na fertilidade do solo com reduçáo da acidez e aumento nos teores de alguns nutrientes em comparação aos solos com vegetação nativa.

Não se verifica diferença nos teores totais de carbono orgânico e das frações húmicas nos solos entre os diferentes usos e coberturas. Há, porém, diferença na qualidade do material orgânico, pois os solos com cultura perene possuem maior relação EA/HUM em comparação com os solos de pastagem e menores teores de carbono não humificado (matéria orgânica leve) em comparação com as áreas de vegetação nativa e pastagem. Todos os solos estudados têm predomínio da fração HUM e baixo grau de humificação da matéria orgânica, mostrando que, independentemente do manejo, as condiçóes naturais da área de estudo favorecem a mineralização da matéria orgânica.

Nas áreas de pastagem verifica-se aumento da densidade do solo e redução da porosidade total, resultantes do pisoteio animal.

\section{REFERÊNCIAS}

ALVES, D.S.; PEREIRA, J.L. G.; SOUSA, C.L. DOS; SOARES, J.V.; YAMAGUCHI, F. Characterizing landscape changes in Central Rondônia using Landsat TM imagery. International Journal of Remote Sensing, v.39, p.2877-2882. 1999. 
ANJOS, J.T.; UBERTI, A.A.A.; VIZZOTO, V.T.; LEITE, G.B.; KRIEGER, M. Physical properties in soils under different management systems. Revista Brasileira de Ciência do Solo, v.18, p.139-145, 1994.

BATISTELLA, M.; MORAN, E.F. Human Dimensions of Land Use and Land Cover in the Amazon: a Contribution from LBA. Acta Amazonica, v.35, p.239-247, 2005.

BRONDIZIO, E.S.; MCCRACKEN, S.D.; MORAN, E.F.; SIQUEIRA, A.D.; NELSON, D.R.; RODRIGUEZ-PEDRAZA, C. The colonist footprint: towards a conceptual framework of deforestation trajectories among small farmers in Frontier Amazônia. In: WOOD, C.; PORRO, R. (Ed.). Land Use and Deforestation in the Amazon. Gainesville: University of Florida, USA, 2002. p.133-161.

BENITES, V.M.; MADARI, B.; MACHADO, P.L.O.A. Quantitative extraction and fractionation of humic substances in soil: a procedure for low cost. Rio de Janeiro: Embrapa Solos, 2003. 7p. (Comunicado técnico, 16)

BERNOUX, M.; FEIG, B.J.; CERRI, C.C.; GERALDES, A.P.A.; FERNANDES, S.A.P. Soil carbon and nitrogen in a forest - pasture chronosequence from Paragominas. Scientia Agricola, v.56, p.777$783,1999$.

CAMARGO, F.A.O.; SANTOS, G.A.; GUERRA, J.G.M. Macromolecules and humic substances, In: SANTOS, G.A.; CAMARGO, F.A.O. (Ed.). Fundamentals of soil organic matter. Genesis: Porto Alegre, 1999. p.27-39.

CANELLAS, L.P.; VELLOSO, A.C.X.; MARCIANO, C.R.; RAMALHO, J.F.G.P.; RUMJANEK, V.M.; REZENDE, C.E. E SANTOS, G.A. Chemical soil properties of an inceptisol under long-term sugarcane crops with vinasse application and without slash burning. Revista Brasileira de Ciência do Solo, v.27, p.935944, 2003.

CERRI, C.C.; VOLKOFF, B. Organic matter from three soils of floodplains in the Marajo/PA. Revista Brasileira de Ciência do Solo, v.12, p.93-100, 1988.

CERRI, C.E.P.; COLEMAN, K.;JENKINSON, D.S.; BERNOUX, M.; VICTORIA, R.; CERRI, C.C. Modeling Soil Carbon from Forest and Pasture Ecosystems of Amazon, Brazil. Soil Science Society of American Journal, v.67, p.1879-1887, 2003.

DABIN, B. The organic matter in tropical soils well drained. Cah. O.R.S.T.O.M., Série Pédologique, v.18, p.197-215 1981.

D’ANDRÉA, A.F.; SILVA, M.L.N.; CURI, N.; GUILHERME, L.R.G. Carbon and nitrogen storage, and inorganic nitrogen forms in a soil under different management systems. Pesquisa Agropecuária Brasileira, v.39, p.179-186, 2004.

EMBRAPA. Centro Nacional de Pesquisa de Solos. Manual of methods for soil analysis. Rio de Janeiro: Embrapa Solos, 1997. $212 \mathrm{p}$.

EMBRAPA. Centro Nacional de Pesquisa de Solos. Brazilian system of soil classification. 2. ed. Rio de Janeiro: Embrapa Solos, 2006. 306p.
FEARNSIDE, P.M. Deforestation in Amazonia: dynamics, impacts and control. Acta Amazonica, v.36, p.395-400, 2006.

FONTANA, A.; PEREIRA, M.G.; LOSS, A.; CUNHA, T.J.F.; SALTON, J.C. Fertility properties and humic fractions in a Rhodic Ferralsol in Brazilian Cerrado. Pesquisa Agropecuária Brasileira, v.41, p.847-853, 2006.

FREIXO, A.A.; MACHADO, P.L.O.A.; GUIMARÃES, C.M.; SILVA, C.A. FADIGAS, F.S. Carbon and nitrogen storage and organic fraction distribution of a cerrado oxisol under diferent cultivation systems. Revista Brasileira de Ciência do Solo, v.26, p.425-434, 2002.

GIAROLA, N.F.B.; TORMENA, C.A.; DUTRA, A.C. Physical degradation of a red latosol used for intensive forage production. Revista Brasileira de Ciência do Solo, v.31, p.863-873, 2007.

GREGO, C.R.; MIRANDA, E.E. DE; VALLADARES, G.S.; CUSTÓDIO, D. DE O.; FRANZIN, J.P.; SILVA, C.F. Exploratory analysis, dynamic space-time production systems in Machadinho (RO) between 1986 and 2005. Campinas: Embrapa Monitoramento por Satélite. 2007. 22p. (Documentos, 64)

LEITE, L.F.C.; MENDONÇA, E.S.; NEVES, J.C.L.; MACHADO, P.L.O.; GALVÃO, J.C.C. Total stocks of organic carbon and its pools in acrisols under forest and under maize cultivated with mineral and organic fertilization. Revista Brasileira de Ciência do Solo, v.27, p.821-832, 2003.

LU, D.; LI, G.; VALLADARES, G.S.; BATISTELLA, M. Mapping soil erosion risk in Rondônia, Brazilian Amazonia: using rusle, remote sensing and gis. Land Degradation and Development, v.15, p.499-512, 2004.

MENDONZA, H.N.S.; LIMA, E.; ANJOS, L.H.C.; SILVA, L.A.; CEDDIA, M.B.; ANTUNES, M.V.M. Chemical and biological properties of a tableland soil cultivated with sugar cane with and without straw burning. Revista Brasileira de Ciência do Solo, v.27, p.201-207, 2000.

MIRANDA, C.C., CANELLAS, L.P.; NASCIMENTO, M.T. Quality of soil organic matter in fragments of atlantic forest and abandoned eucalyptus plantations. Revista Brasileira de Ciência do Solo, p.31, p.905-916, 2007.

MOREIRA, A.; MALAVOLTA, E. Dynamic of organic matter and microbial biomass in a soil under different management systems in the Western Amazon. Pesquisa Agropecuária Brasileira, v.39, p.1103-1110, 2004.

MULlER, M.M.L.; GUIMARÃES, M.F.; DESJARDINS, T.; Martins, P.F.S. Pasture degradation in the Amazon region: soil physical properties and root growth. Pesquisa Agropecuária Brasileira, v.36, p.1409-1418, 2001.

NIMER, E. Climatology of Brazil. 2.ed. Rio de Janeiro: Fundação IBGE, 1989. 421p.

PASSOS, R.R.; RUIZ, H.A.; MENDONÇA, E.S.; CANTARUTTI, R.B.; SOUZA, A.P. Humic substances, microbial activity and labile organic carbon in aggregates of a Dystrophic Red Latosol under two vegetation covers. Revista Brasileira de Ciência do Solo, v.31, p.1119-1129, 2007. 
RANGEL, O. J. P.; SILVA, C.A. Carbon and nitrogen storage and organic fractions in Latosol submitted to different use and management systems. Revista Brasileira de Ciência do Solo, v.31, p.1609-1623, 2007.

RICE, J.A. Humin. Soil Science, v.166, p.848-857, 2001.

ROSALES, M.A.; OLIVEIRA, O.S.; MOURA, M.A.; LOURES, E.G. Influence of organic and mineral fertilization on the characteristics of solid fractions of humic substances in soil. Revista Ceres, v.46, p.67-81, 1999.

SILVA, G.R.; SILVA JUNIOR, M.L.; MELO, V.S. Efeitos de diferentes usos da terra sobre as características químicas de um Latossolo Amarelo do Estado do Pará. Acta Amazonica, v.36, p.151-158, 2006.

SOUZA, Z.M.; LEITE, J.A.; BEUTLER, A.N. Comportamento de atributos físicos de um Latossolo Amarelo sob agroecossistemas do Amazonas. Engenharia Agrícola, v.24, p.654-662, 2004.
TOMÉ JUNIOR, J.B. Manual interpretation of soil analysis. Guaíba: Agropecuária, 1997. 247p.

TORRES, J.L.R.; PEREIRA, M.G.; ANDRIOLI, A.; POLIDORO, J.C.; FABIAN, A.J. Cover crops residue decomposition and nitrogen release in a cerrado soil. Revista Brasileira de Ciência do Solo, v.29, p.606-618, 2005.

VALLADARES, G.S.; BOGNOLA, I.A.; GOUVÊA, J.R.F. Reconnaissance soil survey of medium intensity of Gleba Machadinho, RO. Campinas: Embrapa Monitoramento por Satélite, 2003a. 92p. (Documentos 30)

VALLADARES, G.S.; PEREIRA, M.G.; ANJOS, L.H.C. Phosphate sorption in low activity clay soils. Bragantia, v.62, p.11-118, $2003 \mathrm{~b}$.

VALLADARES, G.S.; DE MARIA, I.C.; QUARTAROLI, C.F.; GREGO, C.R.; CAMARGO, O.A. Index of soil quality in northeastern São Paulo State. Campinas: Embrapa Monitoramento por Satélite, 2007. 17p. (Circular Técnica 13) 